\title{
Efecto inhibitorio del extracto etanólico de Piper aungustifolium (Matico) sobre el crecimiento y desarrollo in vitro de Enterococcus faecalis.
}

\author{
Inhibitory effect of the ethanolic extract of Piper angustifolium (Matico) on the \\ growth and development in vitro of Enterococcus faecalis.
}

\author{
${ }^{1}$ Juan Guillermo Bornaz Acosta, ${ }^{2}$ Zenaida Inofuentes Espinoza, ${ }^{3}$ Soledad Amparo Bornas Acosta, \\ ${ }^{4}$ Denisse Ethiel Tito Aquino y ${ }^{5}$ Vanessa Lissete Bornaz Arenas.
}

\section{RESUMEN}

El Enterococcus faecalis es considerada una de las cepas más responsables de las caries dentales, con gran capacidad de adaptación y tolerancia a las condiciones de un medio adverso, siendo difícil su erradicación.

Palabras Clave: Antimicrobiano, Fracaso Endodóntico, Enterococcus faecalis, Piper aungustifolum, Hidróxido de Calcio.

\begin{abstract}
Enterococcus faecalis is considered one of the strains with great capacity of adaptation and tolerance to an adverse, environmental conditions still difficult its eradication.
\end{abstract}

Keywords: Antimicrobial, Endodontic Failure, Enterococcus faecalis, Piper aungustifolum, Calcium Hydroxide.

Objetivo. El objetivo de esta investigación fue establecer el efecto inhibitorio de la infusión de Piper aungustifolium, (Matico) sobre la cepa Enterococcus faecalis.

Métodos. Se realizó una siembra de la cepa ATCC 29212 (Enterococcus faecalis) en placas con Agar Cerebro Corazón BHI, colocando en la superficie del medio sensidiscos de $5 \mathrm{~mm}$ de diámetro embebidos con las soluciones del Matico, y se hizo 3 pozos de $5 \mathrm{~mm}$ de diámetro por $6 \mathrm{~mm}$ de profundidad, utilizándose una puntera descartable de $1 \mathrm{ml}$, previamente tratada y esterilizada para colocar el Hidróxido de Calcio como grupo control y Piper aungustifolium como grupo experimental, para cada una se utilizó 24 muestras; luego se incubó a 37 oC a las 24, 48, 72 horas y 7 días se tomó medidas.
Resultados. Los promedios de la medida del halo inhibitorio Piper aungustifolum (Matico) fueron menores que la del Hidróxido de Calcio. Se aplicó posteriormente $\mathrm{T}$ de Student $(\mathrm{p}<0.05)$, dando como resultado diferencia estadísticamente significativa entre los datos obtenidos para las tomas a las 24,72 horas y 7 días, exceptuando a las 48 horas donde no hubo diferencia entre ambas sustancias.

\section{INTRODUCCIÓN}

Una de las dolencias más comunes en estomatología es la caries, afectando la pulpa dental, lo cual amerita realizar una endodoncia, consistente en su remoción radical (1). En todo procedimiento de endodoncia, se debe utilizar un medicamento intraconducto principalmente para contribuir con la eliminación de

\footnotetext{
Docente Principal de la Universidad Nacional Jorge Basadre Grohmann E-mail: willybornaz@hotmail.com 2Cirujano Dentista Práctica Privada E-mail: Zenaida.inofuente.aqp@hotmail.com

${ }^{3}$ Docente Principal de la Universidad Nacional Jorge Basadre Grohmann E-mail: solenadbornas1234@hotmail.com

${ }^{4}$ Docente de la Universidad Andina Néstor Cáceres Velásquez E-mail: ethiel19@hotmail.com

5ocente de la Universidad Andina Néstor Cáceres Velásquez E-mail: bornazzz@hotmail.com 
los microorganismos residuales y sus toxinas, luego de la reparación biomecánica (2). Sin embargo, el tratamiento del conducto radicular está asociado de características clínicas relacionadas de manera indirecta con el proceso fisiopatológico de la afección del tejido pulpar, o por los procedimientos terapéuticos $(3,4,5)$.

Enterococus faecalis, es una bacteria en forma de coco dispuesta en cadenas o pares, Gram positiva, anaerobia facultativa, inmóvil y no esporulada que, en años recientes, ha atraído la atención de diversos investigadores porque ha sido identificada como una causa frecuente de infección del sistema de conductos radiculares en dientes con fracaso en el tratamiento endodóntico $(3,5,6)$.

En medicina natural los principios activos de diversas especies de plantas, han demostrado tener múltiples beneficios en comparación a las sustancias sintéticas producidas en laboratorio usadas para procedimientos terapéuticos en estomatología $(1,4,7)$. La Piper aungustifolium es una planta oriunda de América del Sur, más conocida con el nombre de "Matico"; perteneciente la familia de las piperáceas originaria del Perú (8). Fue clasificada por el botánico, recolector, doctor y farmacéutico alemán David Heinrich Hoppe (1760-1846) y sus poderes antimicrobianos han sido reconocidos por la medicina tradicional $(6,7)$.

Las bondades mostradas por el matico en medicina natural motiva, que se realicen investigaciones para determinar su efecto antimicrobiano, para utilizarla como alternativa en los tratamientos de endodoncia frente a las que ya existen en el mercado, donde las sustancias naturales resultan ser las más indicadas, debido que presentan menores efectos adversos $(7,9$, $10,11)$. Estos antecedentes nos han motivado a estudiar el efecto antimicrobiano de la Piper aungustifolium sobre Enterococus faecalis. El objetivo de este trabajo de investigación ha sido determinar el efecto antimicrobiano de infusiones etanolicas de Piper aungustifolium sobre los cultivos de Enterococcus faecals.

\section{MATERIALES Y METODOS}

Tipo de Investigación: longitudinal y prospectivo

Nivel de Investigación: Comparativo Experimental

Diseño: Factorial de Grupos

\section{Identificación de los grupos}

Los grupos serán distribuidos en 16 placas petri, 24 muestras para cada uno de los grupos; grupo experimental: Piper aungustifolium, grupo control: Hidróxido de Calcio.

\section{Criterios Incluyentes}

CepaEnterococcus faecalis

\section{Criterios Excluyentes}

\section{Cepa contaminada de Enterococcus faecalis}

Descripción de la Técnica Se utilizó como materiales: un extracto etanólico de Piper aungustifolium, para el desarrollo del grupo experimental; la cepa ATCC 29212 (Enterococcus faecalis) y una pasta de Hidróxido de Calcio para el grupo control.

La cepa se reactivó de sus culturas originales en $250 \mathrm{ml}$ de infusión caldo cerebro - corazón BHI luego se realizó la siembra en agar cerebro corazón BHI, las cepas de Bornaz et al.

Ciencias, Vol. 3 43-55 (2019)Enterococcus faecalis fueron diluidas para su concentración final de $1 \times 10^{6}$ cels $/ \mathrm{ml}$. para los $500 \mathrm{ml}$. Se dividió la placa en 3 partes para colocar sensidiscos embebidos con las soluciones del Matico, y se hizo 3 pozos de $5 \mathrm{~mm}$ de diámetro por $6 \mathrm{~mm}$ de profundidad, utilizándose una puntera descartable de $1 \mathrm{~mL}$, previamente tratada y esterilizada para colocar el Hidróxido de Calcio, las placas fueron rotuladas y almacenadas en la cámara de anaerobiosis y se incubaron por un lapso 7 días, en los cuales se tomó medidas a las 7, 24, 48 horas y 72 días

\begin{tabular}{|c|c|c|c|}
\hline & Variables & Indicadores & Sub indicadores \\
\hline $\begin{array}{c}\text { Variable } \\
\text { Estímulo } 1 \\
\text { Variable }\end{array}$ & $\begin{array}{l}\text { Piper aungustifolium } \\
\text { (Matico) }\end{array}$ & $\begin{array}{c}\text { Concentració } \mathrm{n} \\
\text { óptima }\end{array}$ & $\mathrm{Mg} / \mathrm{ml}$ \\
\hline $\begin{array}{l}\text { Estímulo } 2 \\
\text { Variable } \\
\text { Respuesta }\end{array}$ & $\begin{array}{l}\text { Hidróxido de calcio } \\
\text { Halo inhibitorio microbiano } \\
\text { de Enterococcus faecalis }\end{array}$ & $\begin{array}{l}\text { Diámetro del } \\
\text { halo inhibitorio }\end{array}$ & $\mathrm{mm}$ \\
\hline
\end{tabular}

Tipo de análisis: Análisis estadístico para muestra pequeña cuantitativa: T Student. 


\section{RESULTADOS}

Tabla $N^{\circ}$ 1. Comportamiento del halo inhibitorio del Enterococcus faecalis por la Piper Aungustifolium (matico)

\begin{tabular}{|c|c|c|c|c|}
\hline \multirow[b]{2}{*}{ Medición } & \multicolumn{4}{|c|}{ Halo (mm.) } \\
\hline & Media & $\begin{array}{c}\text { Desviación } \\
\text { Estándar }\end{array}$ & Mínimo & Máximo \\
\hline 24 horas & 9.03 & 0.38 & 8.50 & 9.75 \\
\hline 48 horas & 9.35 & 0.31 & 8.75 & 9.75 \\
\hline 72 horas & 9.77 & 0.31 & 9.25 & 10.25 \\
\hline 7 días & 9.89 & 0.27 & 9.50 & 10.25 \\
\hline
\end{tabular}

Fuente: Matriz de datos.

$\mathrm{P}=0.000(\mathrm{P}<0.05) \mathrm{S} . \mathrm{S}$

El halo inhibitorio del Enterococcus faecalis por la Piper augustifolium (Matico), a las 24 horas el halo alcanzó un diámetro de $9.03 \mathrm{~mm}$, oscilando entre 8.50 $\mathrm{mm}$ y $9.75 \mathrm{~mm}$; a las 48 horas un valor promedio de $9.35 \mathrm{~mm}$, oscilando entre $8.75 \mathrm{~mm}$ y $9.75 \mathrm{~mm}$; a las 72 horas el valor promedio fue de $9.77 \mathrm{~mm}$, oscilando entre $9.25 \mathrm{~mm}$ y $10.25 \mathrm{~mm}$ y finalmente a los 7 días el halo obtuvo un valor promedio de $9.89 \mathrm{~mm}$, oscilando entre $9.50 \mathrm{~mm}$ y $10.25 \mathrm{~mm}$.

Existen diferencias significativas entre las medias de los halos de inhibición obtenidas a través del tiempo, lo que demuestra que existen cambios en el comportamiento del halo por la Piper aungustifolium (Matico), dado que el efecto aumenta conforme pasa el tiempo.

Figura $\mathrm{N}^{\circ}$ 1. Comportamiento del halo inhibitorio del Enterococcus faecalis por la Piper Aungustifolium (matico)

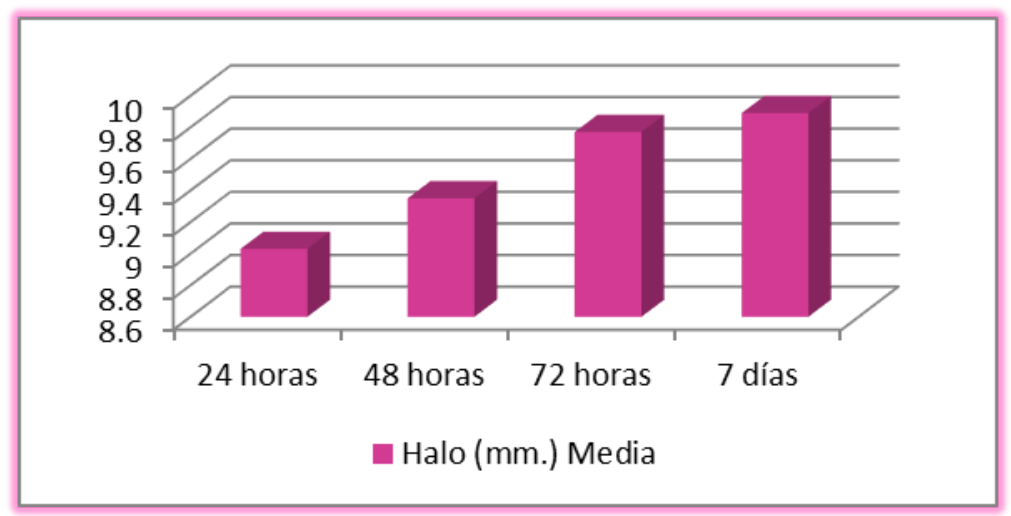

Fuente: Matriz de datos

Tabla N ${ }^{\text {2 }}$. Comportamiento del halo inhibitorio del Enterococcus Faecalis por el hidróxido de calcio.

\begin{tabular}{ccccc} 
Medición & Media & $\begin{array}{c}\text { Halo }(\mathrm{mm} .) \\
\text { Desviación } \\
\text { Estándar }\end{array}$ & Mínimo & Máximo \\
\hline 24 horas & 9.73 & 0.33 & 9.25 & 10.25 \\
48 horas & 9.62 & 0.25 & 9.25 & 10.00 \\
72 horas & 9.58 & 0.22 & 9.25 & 10.00 \\
7 días & 9.65 & 0.17 & 9.50 & 10.00
\end{tabular}

Fuente: Matriz de datos. $\quad \mathrm{P}=0.135(\mathrm{P} \geq 0.05)$ N.S. 
El halo inhibitorio del Enterococcus faecalis por el Hidróxido de Calcio, a las 24 horas el halo alcanzó un diámetro de $9.73 \mathrm{~mm}$, oscilando entre $9.25 \mathrm{~mm}$ y 10.25 $\mathrm{mm}$; a las 48 horas el halo obtuvo un valor promedio de $9.62 \mathrm{~mm}$, oscilando entre $9.25 \mathrm{~mm}$ y $10.00 \mathrm{~mm}$; a las 72 horas el valor promedio observado del halo fue de 9.58 $\mathrm{mm}$, oscilando entre $9.25 \mathrm{~mm}$ y $10.00 \mathrm{~mm}$; y finalmente a los 7 días el halo obtuvo un valor promedio de $9.65 \mathrm{~mm}$, oscilando entre $9.50 \mathrm{~mm} \mathrm{y}$ $10.00 \mathrm{~mm}$.

Según la prueba estadística, no existen diferencias significativas entre las medias de los halos de inhibición obtenidas a través del tiempo, lo que demuestra que no existen cambios en el comportamiento del halo por el Hidróxido de Calcio

Figura $\mathrm{N}^{\circ}$ 2. Comportamiento del halo inhibitorio del Enterococcus faecalis por el hidróxido de calcio.

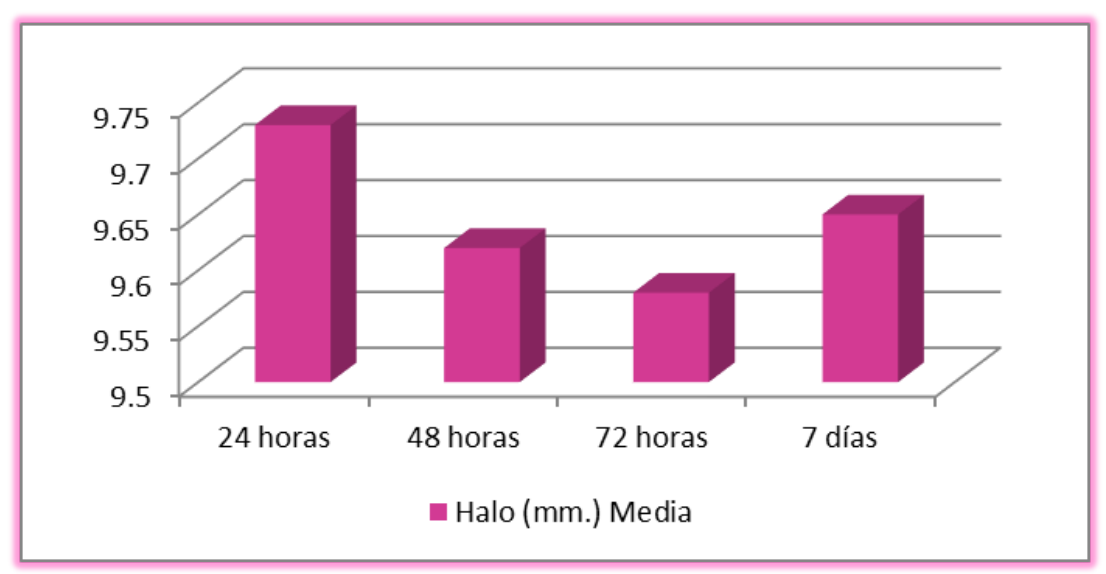

Tabla N ${ }^{0}$ 3. Comparación del halo de inhibición del Enterococcus Faecalis a las 24 horas entre la Piper Aungustifolium (matico) y el hidróxido de calcio.

\begin{tabular}{ccc|}
\hline Halo $(\mathrm{mm})$. & \multicolumn{2}{c}{ Grupo de Estudio } \\
24 horas & Piper & Hidróxido de \\
& Aungustifolium & Calcio \\
Media & 9.03 & 9.73 \\
Desviación Estándar & 0.38 & 0.33 \\
Mínimo & 8.50 & 9.25 \\
Máximo & 9.75 & 10.25 \\
Total & 24 & 24 \\
Fuente : Matriz de datos. & & $\mathrm{P}=0.000(\mathrm{P}<0.05)$ S.S.
\end{tabular}

La comparación, a las 24 horas, entre los halos inhibitorios del Enterococcus Faecalis por la Piper aungustifolium (Matico) y el Hidróxido de Calcio. Para el Piper aungustifolium el halo obtuvo un promedio de $9.03 \mathrm{~mm}$, oscilando entre $8.50 \mathrm{~mm}$ y $9.75 \mathrm{~mm}$; mientras que para el hidróxido de Calcio el promedio llegó a un valor de $9.73 \mathrm{~mm}$, oscilando entre $9.25 \mathrm{~mm}$ y 10.25 mm.

Existe diferencia estadísticamente significativa entre ambos, deduciéndose que el Hidróxido de Calcio es mejor que la Piper aungustifolium (Matico) a las 24 horas. 
Figura $\mathrm{N}^{\circ}$ 3. Comparación del halo de inhibición del Enterococcus Faecalis A las 24 horas entre la Piper Aungustifolium (matico) y el hidróxido de calcio.

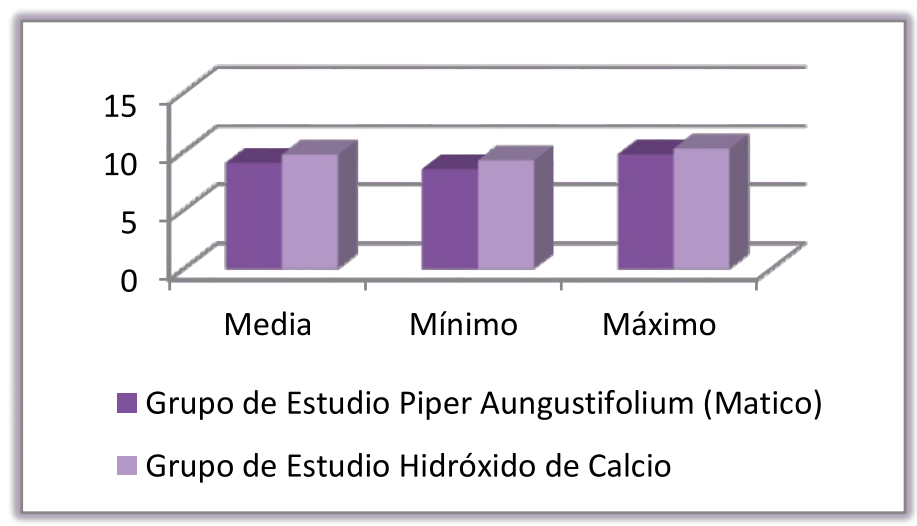

Fuente : Matriz de datos.

Tabla 4. Comparación del halo de inhibición del Enterococcus Faecalis A las 48 horas entre la Piper Aungustifolium (matico) y el hidróxido de calcio.

\begin{tabular}{|ccc|}
\hline Halo $(\mathrm{mm})$. & \multicolumn{2}{c|}{ Grupo de Estudio } \\
48 horas & $\begin{array}{c}\text { Piper } \\
\text { aungustifolium }\end{array}$ & $\begin{array}{c}\text { Hidróxido de } \\
\text { Calcio }\end{array}$ \\
\hline Media & 9.35 & 9.62 \\
Desviación Estándar & 0.31 & 0.25 \\
Mínimo & 8.75 & 9.25 \\
Máximo & 9.75 & 10.00 \\
Total & 24 & 24 \\
Fuente: Matriz de datos & $\mathrm{P}=0.054(\mathrm{P} \geq 0.05)$ N.S. & \\
\hline
\end{tabular}

A las 48 horas se aprecia que para la Piper aungustifolium (Matico) el halo obtuvo un promedio de $9.35 \mathrm{~mm}$, oscilando entre $8.75 \mathrm{~mm}$ y $9.75 \mathrm{~mm}$; mientras que para el Hidróxido de Calcio el promedio llegó a un valor de $9.62 \mathrm{~mm}$, oscilando entre $9.25 \mathrm{~mm}$ y $10.00 \mathrm{~mm}$

Figura $N^{\circ}$ 4. Comparación del halo de inhibición del Enterococcus Faecalis A las 48 horas entre la Piper Aungustifolium (matico) y el hidróxido de calcio.
Según Estadística, las diferencias entre ambos grupos no son significativas, por lo tanto, los grupos son iguales, deduciéndose que el Hidróxido de Calcio y la Piper aungustifolium son igual de efectivos a las 48 horas.

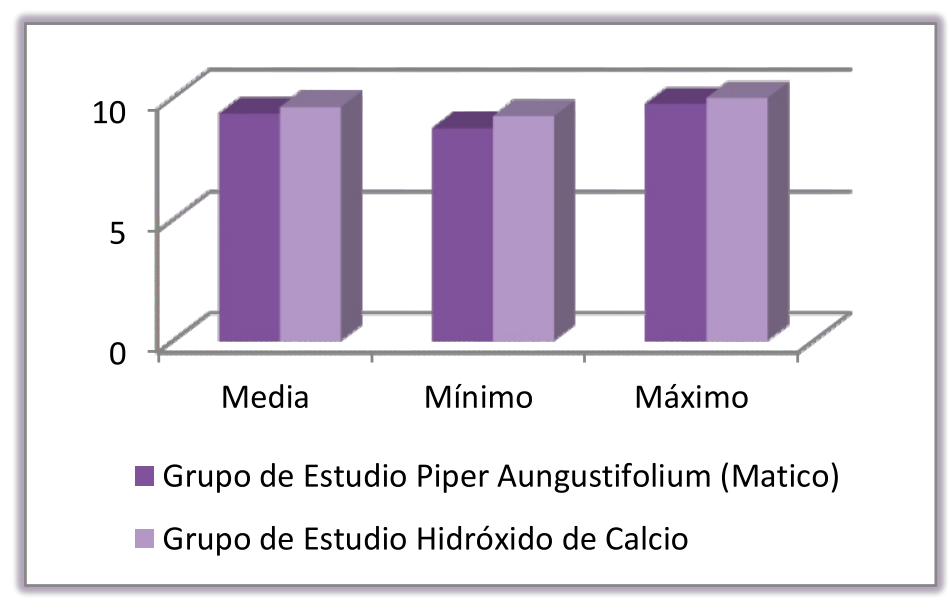

Fuente : Matriz de datos. 
Efecto inhibitorio del extracto etanólico de piper aungustifolium (matico) sobre el crecimiento y desarrollo in vitro de enterococcus faecalis

Tabla N ${ }^{0}$ 5. Comparación del halo de inhibición del Enterococcus Faecalis a las 72 horas entre la Piper Aungustifolium (matico) y el hidróxido de calcio.

\begin{tabular}{ccc|}
\hline Halo $(\mathrm{mm})$. & \multicolumn{2}{c|}{ Grupo de Estudio } \\
72 horas & $\begin{array}{c}\text { Piper } \\
\text { aungustifolium })\end{array}$ & $\begin{array}{c}\text { Hidróxido de } \\
\text { Calcio }\end{array}$ \\
Media & 9.77 & 9.58 \\
Desviación Estándar & 0.31 & 0.22 \\
Mínimo & 9.25 & 9.25 \\
Máximo & 10.25 & 10.00 \\
Total & 24 & 24 \\
Fuente : Matriz de datos & $\mathrm{P}=0.022(\mathrm{P}<0.05)$ S.S.
\end{tabular}

La comparación, a las 72 horas, para el Matico se obtuvo un promedio de $9.77 \mathrm{~mm}$, oscilando entre 9.25 mm y $10.25 \mathrm{~mm}$, mientras que para el Hidróxido de Calcio el promedio llego a un valor de $9.58 \mathrm{~mm}$, oscilando entre $9.25 \mathrm{~mm}$ y $10.00 \mathrm{~mm}$.
Según la Prueba Estadística, las diferencias entre ambos grupos son significativa, por lo tanto, los grupos son diferentes, deduciéndose que la Piper aungustifolium (Matico) es más efectivo que el Hidróxido de Calcio a las 72 horas.

Figura $\mathrm{N}^{\circ}$ 5. Comparación del halo de inhibición del Enterococcus Faecalis a las 72 horas entre la Piper Aungustifolium (matico) y el hidróxido de calcio.

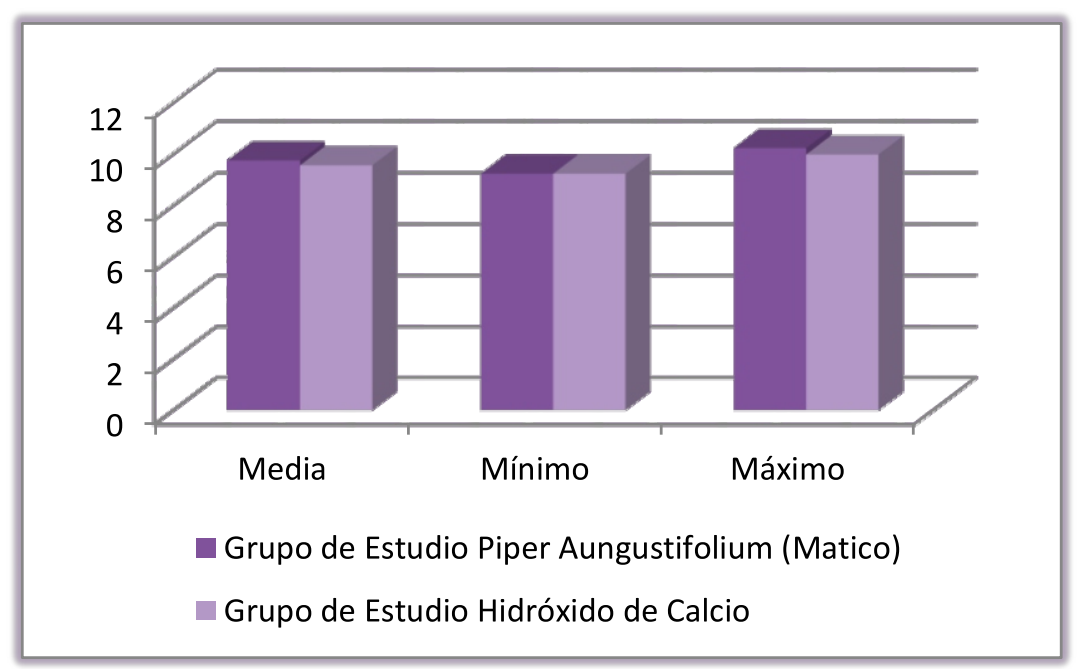

Fuente : Matriz de datos.

Tabla $N^{\text {o }}$ 6. Comparación del halo de inhibición del Enterococcus faecalis a los 7 días entre la Piper Aungustifolium (matico) y el hidróxido de calcio.

\begin{tabular}{|ccc|}
\hline Halo $(\mathrm{mm})$. & \multicolumn{2}{c|}{ Grupo de Estudio } \\
7 días & $\begin{array}{c}\text { Piper } \\
\text { aungustifolium }\end{array}$ & $\begin{array}{c}\text { Hidróxido de } \\
\text { Calcio }\end{array}$ \\
Media & 9.89 & 9.65 \\
Desviación Estándar & 0.27 & 0.17 \\
Mínimo & 9.50 & 9.50 \\
Máximo & 10.25 & 10.00 \\
Total & 24 & 24 \\
Fuente : Matriz de datos & $\mathrm{P}=0.001(\mathrm{P}<0.05) \mathrm{S} . \mathrm{S}$ \\
\hline
\end{tabular}


La comparación, a los 7 días, para la Piper aungustifolium el halo obtuvo un promedio de $9.89 \mathrm{~mm}$., oscilando entre $9.50 \mathrm{~mm}$ y $10.25 \mathrm{~mm}$, mientras que para el Hidróxido de Calcio el promedio llegó a un valor de $9.65 \mathrm{~mm}$, oscilando entre $9.50 \mathrm{~mm}$ y $10.00 \mathrm{~mm}$.
Según la Prueba Estadística, las diferencias entre ambos grupos son significativa, por lo tanto, los grupos son diferentes, deduciéndose que la Piper aungustifolium (Matico) es mejor que el Hidróxido de Calcio a los 7 días.

Figura $\mathrm{N}^{\circ}$ 6. Comparación del halo de inhibición del Enterococcus Faecalis a los 7 días entre la Piper Aungustifolium (matico) y el hidróxido de calcio.

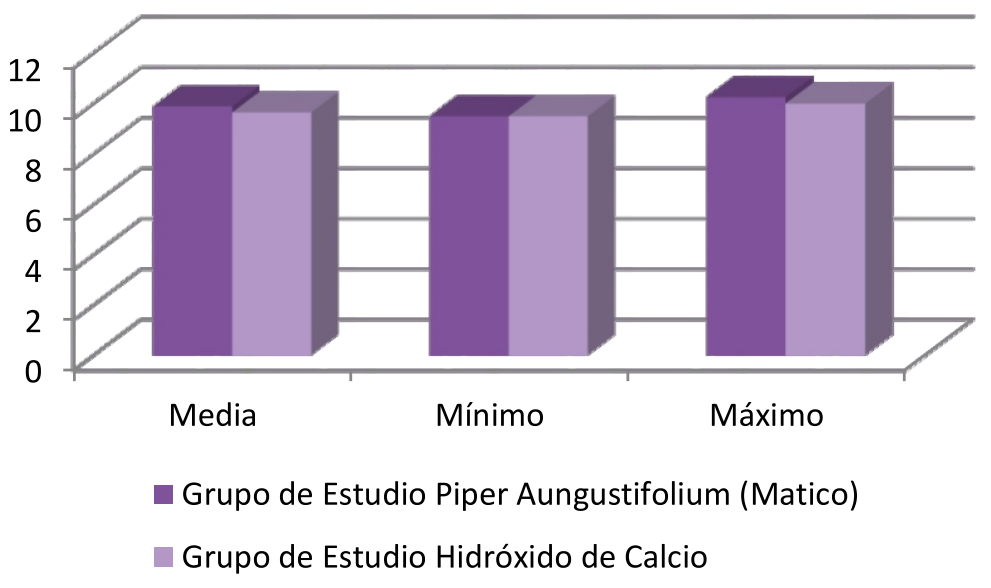

FUENTE: Matriz de datos.

\section{Discusión}

Sánchez Ruiz y col. (13), su investigación sobre actividad microbiana de Hipoclorito de sodio al $5 \%$ en la dilución $1: 1(2.5 \%)$ dio como resultado que la actividad antimicrobiana no duró hasta las 72 horas. Podría deberse a que se trabajó con hipoclorito diluido, por lo tanto, el alcance antimicrobiano se redujo, comparándolo con nuestro estudio el efecto antimicrobiano si duro 72 horas por que se trabajó con hipoclorito al $5 \%$.

Espinel Pinzon Mercy (14), su estudio fue realizado en 30 premolares que fueron infectados con enterococcus Faecalis, a los cuales se les colocó 4 combinaciones de $\mathrm{NaOCl}$ (hipoclorito) y EDTA. Los resultados obtenidos fueron que ninguna de las sustancias utilizadas ya sea solas o mezcladas con el quelante logró eliminar la cepa en cuestión. Uno de los factores que podría haber dado este resultado es que la investigación se realizó en diente natural y su compleja estructura anatómica pudo haber afectado el efecto del hipoclorito, a comparación de los resultados obtenidos en la presente investigación donde sí se apreció un efecto antimicrobiano en la cepa escogida.

Kloucek, Py col. (15), al estudiar el efecto de extractos etanólicos al $80 \%$ de nueve plantas obtenidas por maceración durante 5 días sobre cinco cepas gran positivas y tres grandes negativas, destacando la utilización de las vainas de Caesalpinia. spinosa. Los resultados obtenidos fueron que para el Enterococcus faecalis se observó una CIM de $0.5 \mathrm{mg} / \mathrm{ml}$, para Bacillus cereus de 8 , y de 16 para las demás bacterias, lo cual determina la especificidad y gran poder antimicrobiano de esta sustancia natural, lo que fue corroborado por nuestro estudio.

\section{Conclusiones}

La Caesalpinia espinosa al 60\% logró un halo inhibitorio de $6.33 \mathrm{~mm}$ en promedio con una desviación típica de 0.68 frente al Enterococcus faecalis.

El HClO al 5.25\% logró un halo inhibitorio de $2.82 \mathrm{~mm}$ en promedio con una desviación típica de 0.7 frente al Enterococcus faecalis. Se encontró diferencia estadísticamente significativa entre el efecto antimicrobiano de la Caesalpinia espinosa (Tara) al $60 \%$ y el $\mathrm{HClO}$ al $5.25 \%$ en el halo inhibitorio del Enterococcus faecalis, esto con un margen de error del $5 \%$. La hipótesis nula fue rechazada, debido a que el Halo inhibitorio de la Tara al $60 \%$ obtuvo mayores valores que el hipoclorito de Sodio al 5.25\% por lo que su efecto antimicrobiano fue mayor para nuestra sustancia experimental en el halo inhibitorio del Enterococcus faecalis.

\section{BIBLIOGRAFÍA}

CRAIG J, Bakland L, Sugita E. Microbiología de la Endodoncia y Asepsia en la práctica endodóntica. En: Ingle J, Bakland L, México. editores. Endodoncia. McGraw-Hill Interamericana 2003; p. 63-93.

HAUMAN CH, Love RM. Biocompatibility of dental materials used in contemporary endodontic therapy: a review. Part 1. Intracanal drugs and 
substances. Int Endod J 2003; 36: 75-85.

CHONG Bs, Pitt Ford Tr. The role of intracanal medication in root canal treatment. J Endod 1992; 25:97-06.

BASRANI B, Ghanem A, Tjäderhane L. Physical and chemical properties of chlorhexidine and calcium hydroxidecontaining medications. J Endod. 2004; 30(6):413-7.

MAGUIRE H, Torabinejad M, Kettering JD. The use of aloe vera gel as an intracanal medicament. J of Endod 1996; 22:193

YOLKEN R. Editores. Manual of Clinical Microbiology. American Society for Microbiology 2002: 207-305.

MATUTE Centeno María Elena. Evaluación in vitro del extracto de piper angustifolium (matico) y la clorhexidina como antisépticos bucales. [Trabajo para obtener el título de Profesional de Cirujano Dentista] 2009. Facultad de Odontologia de la Universidad Federico Vila Real. Lima - Perú.

CLAROS, Bilbao, Damiani, Gonzales, Estensoro, Álvarez. Actividad Anti-Helicobacter Pylori de Plantago major, Clinopodium bolivianum, Caléndula officinalis y Piper aungustifolium por el método de difusión de disco. BIOFARBO 1997; Vol. XVMahabir P. Gupta. "270 plantas medicinales iberoamericanas" programa iberoamericano de ciencia y tecnología para el desarrollo. Colombia. Convenio Andrés bello, editorial presencia ltda.1995.
ESTRELA C, Pécora Jd, Souza-Neto Md, Estrela Cr, Bammann 11. Effect of vehicle on antimicrobial properties of calcium hydroxide pastes. Braz dent j. 1999; 10:63-

RODRÍGUEZ-Varo L, Pumarola J, Canalda C. Acción antimicrobiana in vitro de distintas medicaciones sobre Enterococcus faecalis y Actinomyces israelii. Endodoncia 2009; Vol. $27\left(\mathrm{~N}^{\mathrm{o}}\right.$ 1):7-12

ORJALA AJ, Wright AD, Behrends H, Folkers G, Sticher O, Ruegger H, et al. Cytotoxic and antibacterial dihydrochalcones from Piper Aungustifolium. J Nat Prod 1994; 57(1): 1826.

MARTÍNEZ L, Acosta H, Duarte ML. Evaluación in vitro de la actividad antimicrobiana del hidróxido de calcio en dos preparaciones en presencia del enterococcus faecalis. Ustasalud Odontología 2004; 3: 71 - 76.

NAKAJO K. Resistance to acidic and alkaline environments in the endodontic pathogen Enterococcus faecalis. Oral Microbiol Immunol 2006; 21:283-8.

HERRERA DR, Tay LY, Kose-Jr C, Andrade TM, Rezende EC, Kozlowski Jr VA, Santos EB. Efecto antibacteriano del hidróxido de calcio $\mathrm{y}$ iodoformo sobre Enterococcus faecalis y Pseudomonas aeruginosa. Rev Estomatol Herediana. 2008; 18(1):5-8. 